\title{
Study on Accuracy of Gough P.S and Guo Stress Formula in Calculating Propellant Bed Stress under Quasi-static Compaction
}

\author{
Yu-ling Zhang , Guo-qing Liu, Jin-ming Li, Guo-dong Wang \\ Ordnance Engineering College, 050003Shijiazhuang, China
}

\begin{abstract}
In order to verify Gough P.S and Guo stress formula and its accuracy in calculating propellant bed stress under quasi-static compaction, the paper adopted electronic universal testing machine to perform quasi-static compaction on propellant beds of two different diameters, and based on experimental data, the paper obtained stresses of the two beds in the process of compression. The paper used stress formula to calculate stresses of the two propellant beds respectively, based on the propellant bed feed and experimental configuration, and the results show that, during initial propellant compaction phase, the calculations are greater than experimental results, when it comes to certain compaction phase, the calculations of the formula are smaller than experimental results; the calculations of the formula are slightly smaller than experimental results.
\end{abstract}

\section{Introduction}

In order to accomplish tactical indicators of high muzzle velocity and gun power, the gun loading density and ballistic pressure become higher and higher, ballistic combustion and mechanical environment become more and more terrible, and recent years have seen accidents of burst barrels and barrel damages, and the safety issue of gun launch becomes more and more prominent ${ }^{[1-3]}$. Researches carried out both at home and abroad show that, the root cause for burst barrel is the propellant fragmentation and increased burning area resulted from extrusion and collision during gun launch, where the propellant bed, under the impact of ballistic gas of high pressure and high velocity within the bore, generates extrusive stress among propellant grains, causing propellant bed to crush shell bottom or bore wall at high speed. When the forces sustained by propellant grains exceed their own mechanical strengths, fragmentation or deformation occur, leading to the increased burning area of propellant charge and abrupt increase in ballistic pressure, and then burst barrel [4-5]. Hence, the quantitative description of propellant extrusion stress during gun launch will be of significance, on the one hand, predictions of extrusion stress could be used to assess whether deformation or fragmentation of propellant charge could happen, and improvement on gun or propellant charge could be made according to the predictions to avoid burst barrels; on the other hand, quantitative descriptions could be used to obtain factors affecting values of extrusion stress, and the incidence of the oversized extrusion stress could be avoided by means of altering initial feeding conditions or others. Moreover, formula of granular extrusion stress is an important substituting formula in two-phase interior ballistic equation, and its accuracy will extremely affect calculations of interior ballistic two-phase equations. If inaccurate, stress formula will cause rather big error in predictions of bore pressure and other ballistic parameters, therefore the accuracy of granular extrusion stress formula must be ensured as much as possible. Currently, the granular stress formulas used in calculating interior ballistic two-phase flow equation are mainly stress formula and stress formula, while the literature points out the approach of obtaining granular stress formula through quasi-static compaction test, and the paper focused on validating stress formula and its accuracy in calculating propellant bed stress under quasi-static compaction.

\section{Test}

\subsection{Testing device and principle}

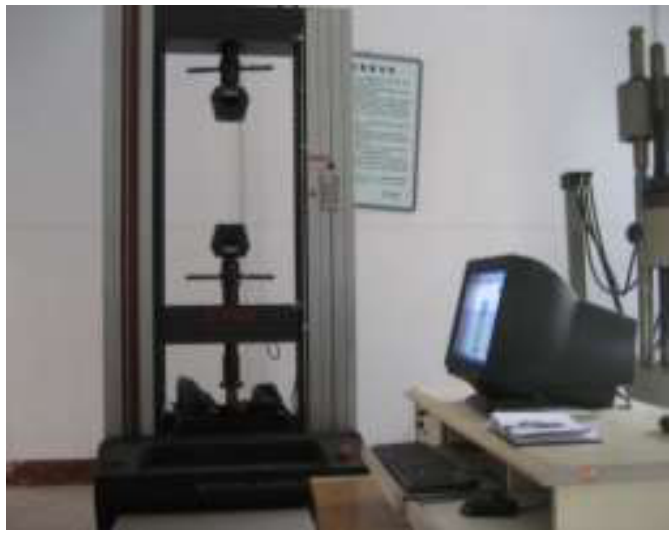

Figure 1. Electronic universal testing machine. 
This paper adopted electronic universal testing machine manufactured by Shenzhen New Sans Materials Co., Ltd, to perform quasi-static compaction on propellant bed, and the testing machine and relevant data capturing equipment are indicated in Fig. 1. In the testing process, data capturing system can record corresponding values of compaction load and compaction displacement.

Table 1. Charged condition of the two propellant beds.

\begin{tabular}{|c|c|c|c|}
\hline \multicolumn{2}{|c|}{ propellant bed Serial No } & $\mathbf{1}$ & $\mathbf{2}$ \\
\hline \multirow{3}{*}{ propellant charge } & quality & $63.5 \mathrm{~g}$ & $103.8 \mathrm{~g}$ \\
\cline { 2 - 4 } & density & $1.6 \mathrm{~g} / \mathrm{cm}^{3}$ & $1.6 \mathrm{~g} / \mathrm{cm}^{3}$ \\
\cline { 2 - 4 } & model & $11 / 7$ & $11 / 7$ \\
\cline { 2 - 4 } & height & $6 \mathrm{~cm}$ & $6 \mathrm{~cm}$ \\
\hline \multirow{2}{*}{ propellant cylinder } & materials & $304 \mathrm{steel}$ & $304 \mathrm{steel}$ \\
\cline { 2 - 4 } & bore & $4.1 \mathrm{~cm}$ & $5.1 \mathrm{~cm}$ \\
\hline \multirow{2}{*}{ piston } & materials & $304 \mathrm{steel}$ & $304 \mathrm{steel}$ \\
\cline { 2 - 4 } & diameter & $4 \mathrm{~cm}$ & $5 \mathrm{~cm}$ \\
\hline
\end{tabular}

\subsection{Test results and analysis.}

From experimental data, we obtain by direct simulation the compressive displacement-pressure curve of propellant bed, and Fig. 2 indicates compressive displacement-pressure curve of the two propellant beds.

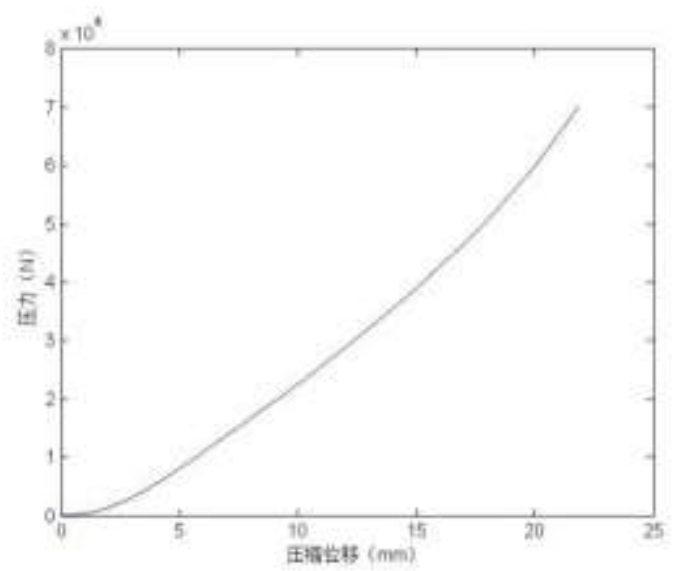

(a) First propellant bed

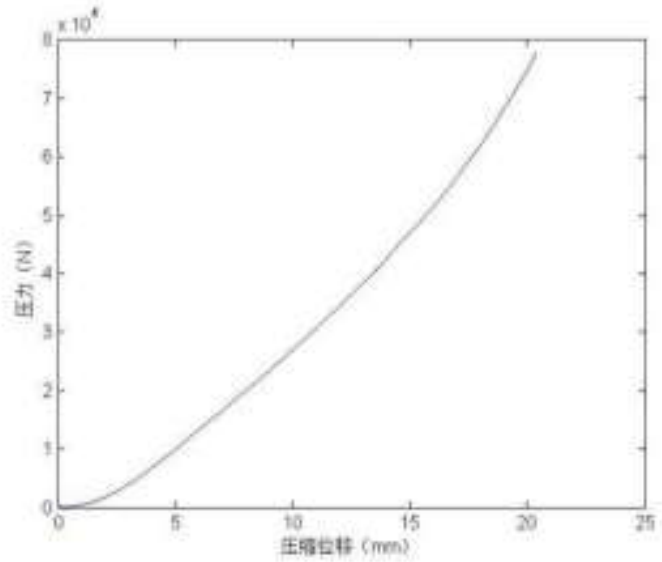

(b) Second propellant bed

Figure 2. Propellant bed Compressive displacement-stress curve.

According to definition of stress, granular extrusion stress of propellant bed at any time is given:

$$
\sigma(t)=\frac{F(t)}{S(t)}
$$

$F(t)$ is pressure at any time, $S(t)$ is extrusion area of propellant bed at any time, and assuming void ratio of propellant bed is evenly distributed, the area occupied by cross section grains of propellant bed is proportional to void ratio, i.e.:

$$
S(t)=S_{0}(1-\phi(t))
$$

where $\mathrm{S}_{0}$ is the cross section area of propellant cylinder.

$$
\phi(t)=\frac{\omega}{\rho_{p} S_{0}\left(H_{0}-V t\right)}
$$

where $\omega$ is the mass of propellant charge, $\rho_{p}$ is propellant charge density, $H_{0}$ is initial height of propellant bed, $V$ is compaction velocity. Substituting known data, we obtain void ratio-granular extrusion stress curve.

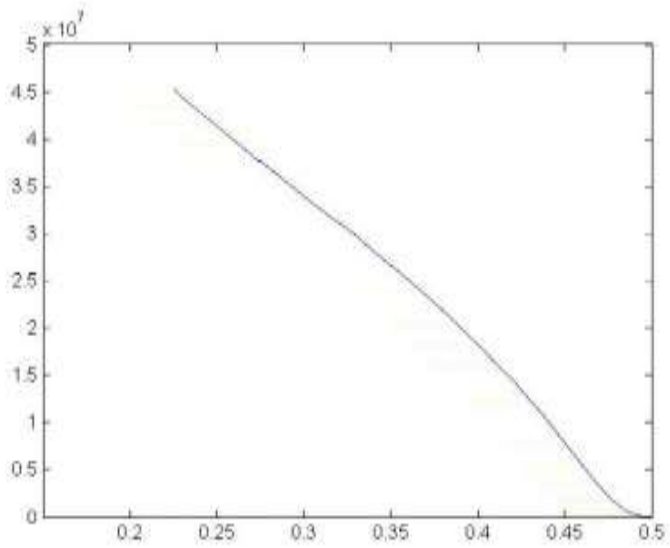

(a) First propellant bed

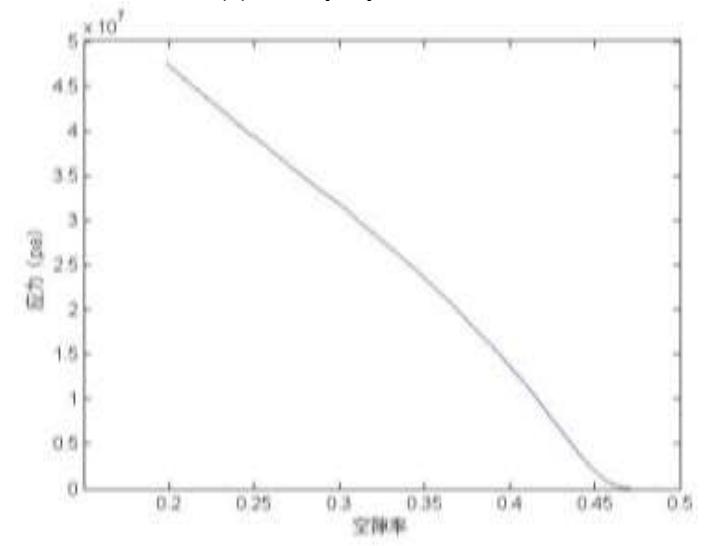

(b) Second propellant bed

Figure 3. Void ratio-stress curve of the first propellant bed.

\section{Calculations of Gough P.S stress formula}

\subsection{Stress formula}


Gough, P. S. based on the similar concept of propagation velocity of weak gas-phase perturbation, adopted equations of momentum and continuity to deduce sound speed formula of solid phase particle group.

$$
a(\phi)=\sqrt{-\frac{1}{\rho_{p}} \frac{d[R(\phi)(1-\phi)]}{d \phi}}
$$

The sound formula of propellant bed with different void ratios could be calculated with following formula:

$$
a(\phi)=\left\{\begin{array}{l}
a_{1}\left(\phi_{0} / \phi\right) \ldots \ldots \ldots \ldots \ldots \ldots \ldots \phi_{0} \\
a_{1} \exp \left[-K\left(\phi-\phi_{0}\right)\right] \ldots \phi_{0}<\phi \leq \phi_{*} \\
0 \ldots \ldots \ldots \ldots \ldots \ldots \ldots \ldots \ldots . . .
\end{array}\right.
$$

where $\phi_{0}$ is compact void ratio, i.e. the void ratio value at the untamped state of propellant grains. $K$ is stress attenuation factor, while $\phi_{*}$ represents the value when void ratio is greater than the value. The relation of $\phi_{0}$ and $\phi_{*}$ could be given: $\phi_{*}=\phi_{0}+0.153 . a_{1}$ is the value of $a$ when $\phi=\phi_{0}$.

Based on the above conclusion, the granular stress formula obtained is written:

$$
R=\left\{\begin{array}{l}
-\frac{\phi-\phi_{0}}{1-\phi} \cdot \frac{\phi}{\phi_{0}} \rho_{p} a^{2} \ldots \ldots \ldots \ldots \ldots \ldots . . . . . . \\
\frac{1}{2 K(1-\phi)} \cdot\left[1-e^{-2 K\left(\phi-\phi_{0}\right)}\right] \rho_{p} a^{2} \ldots \phi_{0}<\phi \leq \phi_{*}
\end{array}\right.
$$

where $\phi_{0}$ is the critical void ratio of untamped propellant charge, $\rho_{p}$ is propellant charge density, $K$ is stress attenuation factor, assume $K=45, a_{1}$ is solid phase sound speed when $\phi=\phi_{0}$, let $a_{1} \approx 426.7 \mathrm{~m} / \mathrm{s}$, where $a_{1}$ is obtained by Soper through measuring American NACO explosive, and at first he measured by certain testing means the average elastic modulus of NACO explosive, and then based on elastic wave theory, used formula $a=\sqrt{E / \rho}$ to obtain sound speed of propellant bed, where $a$ is propellant bed velocity, and $\rho$ is propellant bed density. At the present time, all $a_{1}$ used in formula in literature are taken approximately as $426.7 \mathrm{~m} / \mathrm{s}$.

The formula is the stress relationship obtained according to propagation theory of small-perturbation in elastic medium, and in the actual process of gun launch, only small portion of propellant charge compaction belongs to elastic deformation, as compaction continues, the propellant charge enters into stages of viscoelasticityplastic deformation and plastic deformation.

\subsection{Test results and analysis}

Substituting know data into formula (6) we obtain as indicated in Fig. 4 void ratio-stress curves of the propellant beds in the test and calculation.

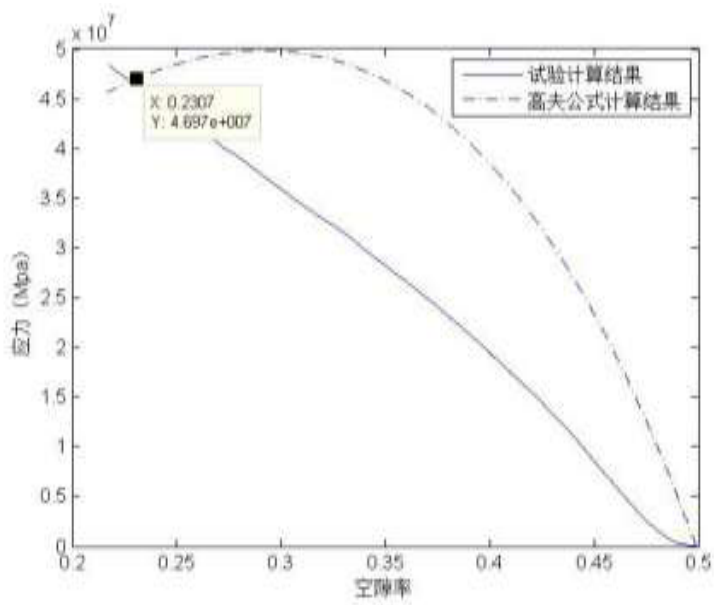

(a) First propellant bed

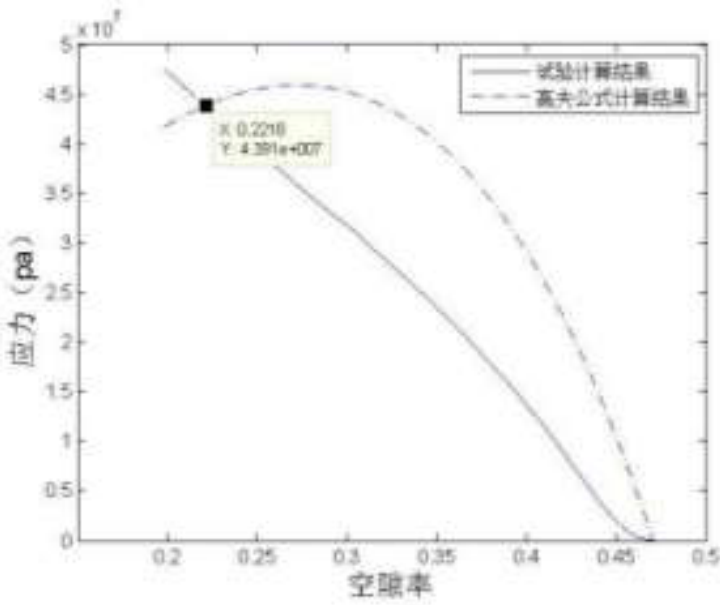

(b) Second propellant bed

Figure 4. Void ratio-stress curves of the propellant bed.

(1) Analyzing Fig. 4, we obtain that in the first propellant bed when void ratio is $(0.2307 \leq \phi \leq 0.499)$, formula calculations are greater than experimental results; when void ratio is 0.2307 , formula calculations are equal to experimental results; within the scope of void ratio $(0.2104 \leq \phi \leq 0.2307)$, experimental results are greater than formula calculations. Analyzing Fig. 7, we obtain that in the second propellant bed when void ratio is $(0.2218 \leq \phi \leq 0.4707)$, formula calculations are greater than experimental results; when void ratio is 0.2218 , the results are equal; within the scope of void ratio $(0.1968 \leq \phi \leq 0.2218)$, experimental results are greater than formula calculations.

(2) Analyzing Fig. 4, note that formula calculations at initial compaction stage are greater than testing results, when it comes to certain compaction stage, testing results are greater than formula calculations, which is because at the initial compaction stage the propellant bed belongs to elastic deformation, both initial void ratios of the first and 
the second propellant beds are greater than 0.4 , sound speed of propellant bed should be smaller than $426.7 \mathrm{~m} / \mathrm{s}$, while the sound speed used in the formula is $426.7 \mathrm{~m} / \mathrm{s}$, such that formula calculations are greater than experimental results; as compaction continues, the propellant bed enters into the stages of viscoelasticplastic deformation and plastic deformation, causing formula calculations to be smaller than experimental results.

(3) Analyzing Fig. 4, obtain that formula calculations of the second propellant bed is more close to experimental results than those of the first propellant bed. One of the causes for such discrepancies between formula calculations and experimental results is sound speed variation of propellant bed under different charged conditions, currently, when applying formula (6), directly assume $a_{1}$ is $426.7 \mathrm{~m} / \mathrm{s}$, which is the sound speed value obtained when void ratio of propellant bed under untamped condition is around 0.4 , and if applied to propellant bed of other charged conditions, it must cause calculation errors. The bigger the difference between initial void ratio of propellant bed and 0.4 , the bigger the calculation errors will be, and the void ratio of the first propellant bed is 0.4990, and that of the second propellant bed is 0.4707 which have well explained that the calculations of the second propellant bed by the formula is closer to the experimental results.

\section{Calculations of Guo stress formula}

\subsection{Stress formula}

Guo, K. K. and others adopted a device specialized for compressing propellant bed to measure its granular stress, and used a compressor with maximum load $5400 \mathrm{~N}$, compression rate $600 \mathrm{~N} / \mathrm{S}$ as an external force for the propellant bed to be loaded. The experimental results measured from propellant charges of different sizes are indicated in Fig. 2, where $K u$ is called compressive parameter of the dimension one, i.e.

$$
K u=\sqrt{\frac{\phi_{0}-\phi}{\phi_{0}}} \phi_{0}^{0.75}
$$

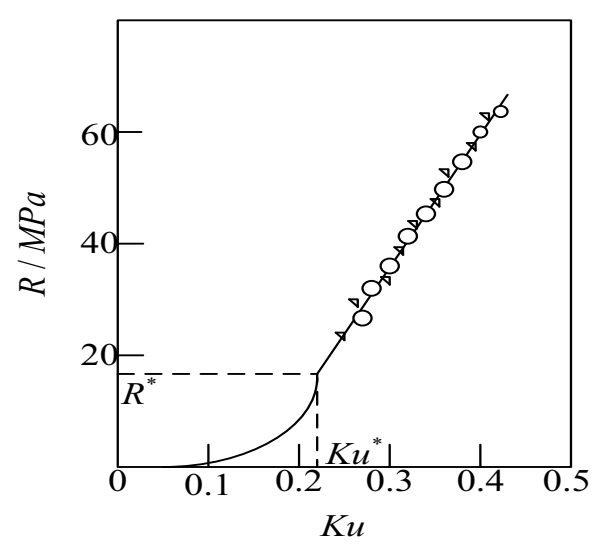

Figure 5. Relationship of $K u$ and granular stress.
It is related to the shape and size of propellant charges, if the size of propellant is small, the initial charged density will be big, such that $\phi_{0}$ will be small, and conversely, it will be big. From Fig. 5, note that at $K u=K u^{*}$, there is a turning point. Soper, W. G and Eaton believed that it is caused by property variations of propellant bed in the process of compaction. After propellant grains are compressed to some extent, called even viscoelastic medium, remarkable changes have taken place in the mechanical performance of the whole propellant bed, leading to incidence of such phenomenon of turning point. As for AmericanWC-870 explosive, the measured relationship of granular stress and $K u$ is given as follows:

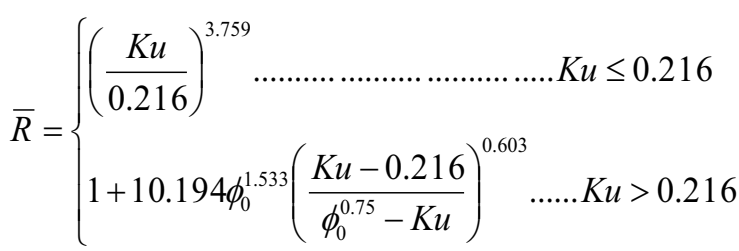

where $\bar{R}=R / R^{*}, R^{*}=14.5 \mathrm{MPa}, K u^{*}=0.216$.

The formula could describe the whole process of propellant bed under quasi-static compaction, from grains filling void, elastic deformation of propellant bed as well as to viscoelasticity-plastic deformation and plastic deformation stages.

\subsection{Test results and analysis}

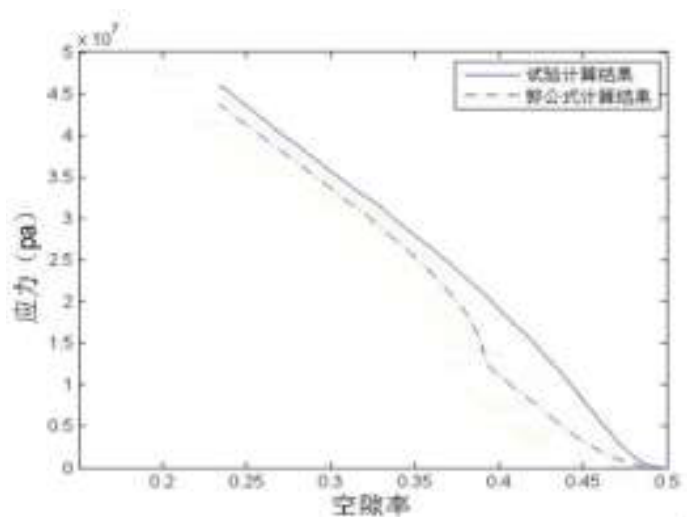

(a) First propellant bed

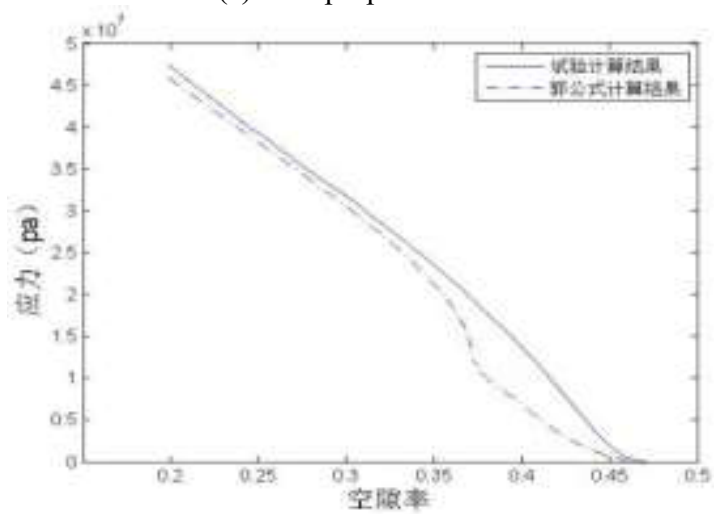

(b) Second propellant bed

Figure 6. The void ratio-stress curves in the test and calculation of the propellant bed. 
Take formula (8) as an example for calculation, substituting the known data into formula (8) to calculate, the calculations of the propellant bed is indicated as Fig. 6.

(1) Analyzing Fig. 6, note that in the first and the second propellant beds, formula calculations are slightly smaller than the experiment results.

(2) By calculation, we obtain that formula calculations are slightly smaller than the testing results, but the difference is not too much, and it is concluded that the formula is capable of describing the quasi-static compaction process accurately, which is because the stress formula could describe accurately the whole process of propellant bed under quasi-static compaction, from stages of propellant grains filling void, elastic deformation as well as to viscoelasticity-plastic deformation and plastic deformation.

\section{Conclusion}

By comparing formula calculations to the experimental results, we obtain that formula calculations are greater than experimental results at the initial compressive stage, when it comes to certain compaction stage, the experimental results are greater than formula calculations, which is mainly because the formula is used to describe elastic deformation, while elastic deformation of propellant bed only happens at the initial stage, and as the compaction goes on, the propellant bed will enter into stages of viscoelasticity-plastic deformation and plastic deformation; Moreover, if the initial sound speed of the propellant bed in the formula $(426.7 \mathrm{~m} / \mathrm{s})$ is used for propellant beds with void ratio far greater than 0.4 , it may cause some calculation errors. The formula could fail to accurately describe propellant stress under quasi-static compaction against the testing configuration.

\section{References}

1. X.T. Rui, L.F. Yun and N.S. Sha: Acta Armamentarii, Vol. 26 (2005) No.5, p.690.

2. X.T. Rui, B.B. Feng and G.P. Wang: Ordnance Industry Automation, Vol. 30 (2011) No.5, p. 56.

3. J.Y. Yang, Y.X. Yuan and X.B. Zhang: Journal of Ballistic, Vol. 11 (1999) No.4, p. 92.

4. X.T. Rui, L.F. Yun and G.P. Wang: Direction to Launch Safety of Ammunition (National Defense Industry Press, Beijing 2009).

5. L.F. Yun: Study of the Method Evaluating the Launch Safety for Gun Propellant Charge (Ph.D., Nanjing University of Science and Technology, China 2008). 